\title{
Familial atypical multiple mole-melanoma (FAMMM) syndrome: segregation analysis
}

\author{
HENRY T LYNCH*, RAMON M FUSARO†, WILLIAM J KIMBERLING+, \\ JANE F LYNCH*, AND B SHANNON DANES§ \\ From * the Department of Preventive Medicine/Public Health and $\dagger$ the Department of Dermatology, \\ Creighton University School of Medicine, Omaha; Nebraska; the Department of Otolaryngology, \\ Boys Town Institute for Hearing and Speech Disorders; and \$the Department of Medicine, \\ Cornell University Medical College, New York, USA.
}

SUMMARY Genetic analysis was performed on four kindreds with clinical and pathological $\stackrel{\omega}{N}$ verification of the FAMMM syndrome. There were 80 affected or at risk members in these families. 을 A segregation ratio of 0.47 was observed, which is consistent with an autosomal dominant mode of $\vec{O}$ inheritance. Three obligate gene carriers who lacked any FAMMM phenotypic manifestations were $\bigcirc$ observed and the rate of penetrance for the FAMMM gene was calculated to be 0.93 . Cancer at all $\stackrel{\circ}{\square}$ anatomical sites (exclusive of cutaneous malignant melanoma and intraocular malignant melanoma) $\stackrel{\mathbb{D}}{-}$ showed a five-fold increase $(\mathrm{p}<0.004)$ in risk for gene carriers when age corrected and compared to $\vec{\oplus}$ the population expectation. Although there was an apparent excess of carcinoma of the lung, $\underset{\omega}{\infty}$ pancreas, and breast, the number of family members studied with specific organ cancer was too small; therefore, a larger sample size will be needed to verify this apparent excess. Our findings warrant further investigation in additional FAMMM kindreds.

In previous papers, ${ }^{1-6}$ we have reported four pedigrees of the familial atypical multiple molemelanoma (FAMMM) syndrome. Even though each of the pedigrees has been analysed separately, phenotypic variability with respect to cutaneous malignant melanoma (CMM) and other cancer occurrences has been documented within each kindred. By analysing all of our cumulative FAMMM data, we are now able for the first time to examine the natural history and test genetic hypotheses of the FAMMM syndrome.

Our purpose is to provide the results of a genetic analysis performed on four informative FAMMM kindreds. These results are preliminary and must be considered with caution. They do however provide support for the hypothesis dealing with variable expressivity and penetrance of the FAMMM gene. Greater precision of this conviction will depend upon recognition of biomarker association and appropriate gene linkage investigations.

Materials, methods, and results

Four kindreds with clinical-pathological verification

Received for publication 20 October 1982. Accepted for publication 5 February 1983. of the FAMMM phenotype in multiple family members have been under our investigation for as long as 16 years. $^{1-6}$ There are 80 affected or at risk members in these families. Ascertainment of these FAMMM kindreds was made through physician referral or diagnosis of the cutaneous phenotype in probands or both by one of the authors.

A genetic analysis was done to determine the frequency of the FAMMM syndrome among offspring at risk. Using a conservative approach for our analysis, we eliminated all of the probands and their affected parents in the direct line of 'ascent'. Thus, eight persons were excluded, leaving 72 informative family members who could be included in the genetic analysis. A person was considered $N$ affected if he/she had CMM or pathologically $N$ verified atypical moles or both. ${ }^{2}{ }^{3}$ Over all, 34 out of $\stackrel{\mathcal{N}}{\mathrm{N}}$ $72(47 \%)$ of the scorable family members conformed $\omega$ to these criteria (table 1). No sex differences relative to risk, age of onset, or severity of phenotype were observed. Furthermore, no heterogeneity between families with regard to these same parameters was observed, although the sample is as yet too limited to preclude the possibility of heterogeneity. Consequently, all analyses were done assuming a homogeneous age of onset distribution between all 
TABLE 1 Frequency of affected by age group.

\begin{tabular}{llrl}
\hline Age & Affected & Normal & Total \\
\hline 0-20 years & 2 & 6 & 8 \\
$21-50$ years & 21 & 23 & 44 \\
51 years & 11 & 9 & 20 \\
Total & $34^{*}$ & 38 & 72 \\
\hline
\end{tabular}

* $\chi^{2}$ test with one degree of freedom was used and the result was not significantly different from $1 / 2$.

Segregation ratio $=34 / 72=0.47$

TABLE 2 Cancer table ( $n=42$ gene carriers).

\begin{tabular}{lccl}
\hline & $\begin{array}{l}\text { No of } \\
\text { patients }\end{array}$ & $\begin{array}{l}\text { Observed } \\
(\%)\end{array}$ & $\begin{array}{l}\text { Expected } \\
(\%)(\text { age } \\
\text { adjusted })\end{array}$ \\
\hline CMM & 21 & $50 \cdot 0$ & - \\
Atypical naevi & 23 & $54 \cdot 8$ & - \\
No CMM or naevi & 3 & $7 \cdot 1$ & - \\
Lung & 3 & $7 \cdot 1$ & $0 \cdot 8(\mathrm{p}<0 \cdot 02)^{*}$ \\
Pancreas & 2 & $4 \cdot 8$ & $0 \cdot 2(\mathrm{p}<0 \cdot 02)^{*}$ \\
Breast & 2 & $4 \cdot 8$ & $0.6(\mathrm{p}<0 \cdot 04)^{*}$ \\
Prostate & 1 & $2 \cdot 4$ & - \\
Tonsil & 1 & 2.4 & - \\
Larynx & 1 & 2.4 & - \\
All non-CMM cancer & $9 \dagger$ & 21.4 & $4 \cdot 1(\mathrm{p}<0 \cdot 004)^{*}$ \\
$\quad$ ('all sites') & & & \\
\hline
\end{tabular}

* Exact probability of $\mathrm{x}$ or more affected calculated using binomial expansion.

tOne patient had both lung and prostate cancer.

four families. Male-to-male transmission observed in these pedigrees excluded $\mathrm{X}$ linkage.

Expressivity of the FAMMM gene with respect to various phenotypic effects among identified and obligate gene carriers was determined (table 2). For the determination of the phenotypic associations, the total sample of 42 affected family members was used. Those eight family members who were discarded in the previous analysis were therefore included. CMM occurred in $50 \%$ and atypical moles in $54 \%$ of the gene carriers. Because CMM or pathologically verified atypical moles or both were used as the definition of an affected family member, these estimates are severely biased and represent maximal frequencies. These results do, however, show the variation in the phenotype and underscore the unreliability of complete dependence upon the presence of both of these physical findings in a single patient when attempting to establish the diagnosis of the FAMMM syndrome. Three subjects $(7.1 \%)$ were identified who did not show any manifestation of this disorder, but who were obligate gene carriers on the basis of pedigree analysis (that is, they had affected parents and children). The $7.1 \%$ rate of non-penetrance $(92.9 \%$ penetrance frequency) must be considered minimal, in as much as it would have been impossible to have detected any unaffected gene carrier who had no affected children. The data were insufficient to calculate an age specific penetrance rate and the penetrance rate given here was calculated from the total sample and included family members under the age of 20 years. We would expect that, as more data become available, the penetrance rate would be found to be positively correlated with age.

There appeared to be a strong association of gene carrier status with other neoplasms. This is illustrated in table 2. Gene carriers show a statistically significant increase in the incidence of non-CMM cancers. Thus, nine out of $42(21 \%)$ gene carriers have been affected with a cancer other than malignant melanoma. In addition, there is a less striking but still significant increase in the number of patients with cancers of the lung, pancreas, and breast. The mean age of the sample of affected family members is 38 years. Expected values were calculated from data presented in the Third National Cancer Survey ${ }^{7}$ and were, of course, age adjusted.

\section{Discussion}

These data lend statistical support to our hypothesis that the FAMMM syndrome (a cancer associated genodermatosis) is inherited as an autosomal dominant trait ${ }^{2} 4$ and displays variable expressivity and reduced penetrance. While half of our sample of gene carriers developed CMM, the lifetime risk for CMM could be greater and needs to be calculated from a large sample. Most disturbing is the observation of an increased statistical risk for cancers at other anatomical sites. This finding was not unexpected. Specifically, we have found an increased cancer spectrum in simple inherited cancer and pre-cancer disorders; for example, ataxia telangiectasia, where in addition to lymphoma and leukaemia, sarcomas, squamous cell carcinoma, and epithelial malignancies are encountered. ${ }^{3}$ If corroborated by additional family data, the management of patients affected with the FAMMM syndrome should be expanded to include surveillance for the presence of these other cancers.

We have previously reported a suspected increased tumour spectrum in the FAMMM based upon one informative kindred. ${ }^{4}$ It was therefore of interest that in our four pedigrees, cancer of all anatomical sites combined, excluding CMM and intraocular malignant melanoma (IOM) (table 2), showed a five-fold increase in risk to gene carriers when age corrected and compared to population expectations. Furthermore, it should be emphasised that cancer, other than CMM, was not a criterion for the diagnosis of gene carrier status. There were other family members who did develop cancer, but who were not counted as gene carriers because they lacked the physical findings necessary by our preliminary definition for such a designation. 
An increase in risk for non-CMM cancers at 'all sites' appears to be supported by these data, but the significance of the apparent increased risk of cancer of the lung, breast, and pancreas is not as secure, as the low numbers of patients relative to the numbers of site specific cancers makes one wary of a random sampling effect. Consequently, the results relative to specific sites are tentative; however, the bizarre occurrences of certain cancers are worthy of note.

Within these four pedigrees were examples of remarkable cancer expression. ${ }^{1-6}$ 8-11 $^{\text {The occurrence }}$ of squamous cell carcinoma of the tonsil in a young (29-year-old) female lacking environmental risk factors (non-alcoholic and essentially non-smoking) was of unusual interest. ${ }^{6}$ In addition, she had a previous history of CMM without having any atypical moles. Her father had FAMMM moles and developed CMM at the age of 29. Later, he developed two separate primary cancers (lung and prostate) when aged 53 and 56 years.

One of the FAMMM kindreds, which we studied in Holland, ${ }^{4}$ had a member of the pedigree who had multiple CMMs but later developed (a year apart) two separate primary IOMs. The incidence of CMM and IOM in general population studies has been found to be about 4 in 100000 and 1 in 100000 , respectively. ${ }^{7}$ Hence, the chance of simultaneous but coincidental occurrences of these lesions in the same patient would be practically nil. A review of published reports showed only two FAMMM patients who showed both IOM and CMM ${ }^{4213}$ and only 22 patients with bilateral IOM. ${ }^{13}$

In one of the two families showing correspondence between in vitro hyperdiploidy and gene carrier status, ${ }^{8}$ a single family member who did not show either CMM or atypical moles, and was classified as unaffected in this study, was nevertheless observed to have a significantly increased frequency of hyperdiploidy. Therefore, he could have been presumed to have been a gene carrier. This patient manifested Hodgkin's lymphoma.

Finally, these FAMMM pedigrees show unusual increased survival with multiple primary CMMs. For example, one patient over two decades had nine separate primary CMMs, of which two were nodular Clark's IV lesions. ${ }^{3} 10$

Collectively, these remarkable clinical-pathological-genetic findings in our four FAMMM kindreds indicate a possibly unique host factor mechanism in the natural history of cancer expression. These observations cover a panorama ranging from cancer resistance to precocious expression of cancer, including variable penetrance and expressivity of the phenotype by a single dominant gene with high penetrance. This is pertinent since clinical evaluation of our patients showed clear evidence of variable expressivity of the phenotype, thereby posing diagnostic difficulty, particularly when an isolated case is encountered showing incomplete expression of the FAMMM phenotype. The important lesson from these observations is that we must not view the patient in isolation but rather in the broader context of his genotypic status. The genotypic status can only be evaluated by a detailed pedigree analysis.

This work was supported by Grant No 1297A, Council for Tobacco Research, USA, Inc, the Fraternal Order of Eagles, and by the National Institutes of Health/National Cancer Institute/ National Large Bowel Project CA 15973, and the National Pancreatic Cancer Project CA 25662.

\section{References}

1 Lynch HT, Frichot BC, Lynch PM, Lynch J, Guirgis HA. Family studies of malignant melanoma and associated cancer. Surg Gynecol Obstet 1975;141:517-22.

2 Lynch HT, Fusaro RM, Pester J, Lynch JF. Familial atypical multiple mole melanoma (FAMMM) syndrome: genetic heterogeneity and malignant melanoma. $\mathrm{Br} J$ Cancer 1980;42:58-70.

${ }^{3}$ Lynch HT, Fusaro RM. Cancer-associated genodermatoses. New York: Van Nostrand Reinhold, 1982:559.

4 Lynch HT, Fusaro RM, Pester J, et al. Tumour spectrum in the FAMMM syndrome. Br J Cancer 1981 ;44:552-60.

${ }^{5}$ Frichot BC, Lynch HT, Guirgis HA, Harris RE, Lynch JF. A new cutaneous phenotype in familial malignant melanoma. Lancet 1977 ; i:864-5.

6 Lynch HT, Fusaro RM, Albano WA, Pester J, Kimberling WJ, Lynch JF. Phenotypic variation in the familial atypical multiple mole-melanoma syndrome (FAMMM). J Med Genet $1983 ; 20: 25-9$.

7 Cutler SJ, Young JL. The Third National Cancer Survey: incidence data. NCI Mono 411 Washington: DHEW, 1975.

8 Lynch HT, Fusaro RM, Danes BS, Lynch FJ, Kimberling WJ. A review of hereditary malignant melanoma including biomarkers in the FAMMM. Cancer Genet Cytogenet (in press).

${ }^{9}$ Lynch HT. Cancer genetics. Springfield, Illinois: C C Thomas, 1975:639.

10 Lynch HT, Krush AJ. Heredity and malignant melanoma: implications for early cancer detection. Can Med Assoc J $1968 ; 99: 17-21$.

11 Lynch HT, Frichot BC, Lynch JF. Familial atypical multiple mole-melanoma syndrome. $J$ Med Genet 1978 ; 15:352-6.

12 Bellet RE, Shields JA, Soll DB, Bernardino EA. Primary choroidal and cutaneous melanomas occurring in a patient with the B-K mole syndrome phenotype. Am J Ophthalmol 1980;89:567-70.

13 Oosterhuis JA, Went LN, Lynch HT. Primary choroidal and cutaneous melanomas, bilateral choroidal melanomas, and familial occurrence of melanoma. Br J Ophthalmol $1982 ; 4: 230-3$.

Correspondence and requests for reprints to Professor H T Lynch, Department of Preventive Medicine/Public Health, Creighton University School of Medicine, Omaha, Nebraska 68178, USA. .

$$
\text { 象 }
$$

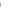
G 